\title{
Further Studies with Lipoamide Dehydrogenase Mutants of Escherichia coli $\mathrm{K} 12$
}

\author{
By J. R. GUEST AND I. T. CREAGHAN \\ Department of Microbiology, University of Sheffield, SIо $2 T N$
}

(Received 20 September 1973)

\begin{abstract}
SUMMARY
The immunological properties of ten lipoamide dehydrogenase mutants of Escherichia coli were investigated with antiserum raised against purified lipoamide dehydrogenase. Seven mutants were CRM + (cross-reacting material present) as they contained lipoamide dehydrogenase proteins exhibiting either complete or partial immunological identity with the wild-type protein. This indicates that at least seven of the mutations affect the lipoamide dehydrogenase structural gene $(l p d)$. The remaining three mutants $(C R M-)$ contained no detectable cross-reacting protein. None of the lpd mutations were sensitive to any of three different amber-suppressors. Genetic analysis by PI-transduction showed that all the $l p d$ mutant sites were clustered very near the distal gene (aceF) of the ace region which specifies the dehydrogenase $(a c e E)$ and transacetylase $(a c e F)$ components of the pyruvate dehydrogenase multienzyme complex. Calculations based on the recombination frequency between an ace $F$ mutant and the nearest lpd mutant site support the conclusion that apart from the possible presence of a regulatory element, the $a c e F$ and $l p d$ genes are contiguous.
\end{abstract}

\section{INTRODUCTION}

The pyruvate and $\alpha$-ketoglutarate dehydrogenase multienzyme complexes of Escherichia coli contain specific dehydrogenase (EIp, Erkg) and transacylase (E2p, E2kg) components but their lipoamide dehydrogenase components $\left(E_{3}\right)$ are functionally interchangeable and indistinguishable by several criteria. Studies with mutants have established the existence of two pairs of closely-linked genes $a c e E$ and $a c e F$ (at $2.3 \mathrm{~min}$ ) and $\operatorname{sucA}$ and $\operatorname{sucB}$ (at 16.3 min) specifying the respective $E_{1}$ and $E_{2}$ components of the pyruvate and $\alpha$-ketoglutarate complexes (Henning \& Hertz, 1964; Herbert \& Guest, 1969). Gene expression in both the ace and suc regions is polarized from the dehydrogenase to the transacylase genes (Henning, Dennert, Hertel \& Shipp, I966; Creaghan \& Guest, 1972) and a regulatory element linked to and controlling expression of the ace genes has been detected (Flatgaard, Hoehn \& Henning, I97I).

Recently mutants deficient in lipoamide dehydrogenase have been isolated and detailed studies with one of these mutants have indicated that $E$. coli possesses a single lipoamide dehydrogenase gene $(l p d)$ specifying the $\mathrm{E}_{3}$ components of both complexes and that this gene is located very close to aceF at the distal end of the ace region (Guest \& Creaghan, 1972; 1973; Guest, 1974).

This paper describes further immunological and genetic studies using a total of ten independent $l p d$ mutants. The results confirm the existence of a single structural gene for lipoamide dehydrogenase and indicate that it is very close to and probably contiguous with the $a c e F$ gene. During the course of this work independent support for these conclusions 
has come from studies with another mutant (sw28; $d h l$ ) which lacks lipoamide dehydrogenase activity (Alwine, Russell \& Murray, 1973).

\section{METHODS}

Bacterial strains. The isolation and biochemical properties of the lipoamide dehydrogenase mutants, T3A58lpdI and 2, ABI325lpd3, XG3lpd4, WGAlpd5, Hlpd6, 7, 8, 9 and Io, and details of the corresponding parental strains have been described previously (Guest \& Creaghan, 1973). Spontaneous streptomycin resistant derivatives of $l p d$ mutants were selected on nutrient agar containing streptomycin sulphate $(200 \mu \mathrm{g} / \mathrm{ml})$. All ten $l p d$ mutations were also introduced into the WGAS (a streptomycin-resistant derivative of wGA; Herbert \& Guest, 1969) background, to provide a series of wGAslpd mutants. To do this Leu transductants or exconjugants were selected from a leucine-requiring derivative of WGAS using $l p d$ donors. The construction of strains T3A58lpdrnadC $(\operatorname{trpE}, \operatorname{trp} A, \operatorname{lpd} I, \operatorname{nadC})$ and AIonadC $(P I)$ (aceFIo, nadC, PI) has been described previously (Guest, 1974). The Hfr and F-prime donors used for mapping were: $\mathrm{H}(\mathrm{Hfr}$, thi $)$ from W. Hayes, BI I ( Hfr, met) and R4 (Hfr, met) from R. H. Pritchard, F $\mathrm{F}_{2}$ gal (=FI53, $\mathrm{gal}^{+}$)/W3Ior ( $\mathrm{galK}$ ) from F. Jacob, KLFI $\left(=\mathrm{FIOI}\right.$, leu $\left.{ }^{+}\right) / \mathrm{AB} 2463$ (leu, pro, his, arg, thi, str) from J. D. Gross and KLF4 (=FIO4, $\left.l e u^{+}\right) / \mathrm{AB} 2463(\arg E, h i s, l e u, t h r, p r o A, r e c A, m t l, x y l$, ara, galK, lac $Y, t s x$, str) from $\mathrm{K}$. B. Low. Suppressibility of the $l p d$ mutations was tested by conjugation with the F-prime donor AW9 (FI4, met $B^{+}$supU)/AWI (metB, rec A56) from J. Scaife and by transduction with derivatives of the $\operatorname{trp}$ deletion strain, w3I Io $\operatorname{trp} R, \operatorname{trp} A-E_{\text {del }}$, carrying $\sup D$ and $\sup F$ mutations.

Growth of organisms, preparation of cell-free extracts and enzyme assay. The media and methods used for the growth of organisms and the preparation of ultrasonic extracts have been described elsewhere (Guest \& Creaghan, 1973). Extracts were stored at $-20{ }^{\circ} \mathrm{C}$. The activities for lipoamide dehydrogenase, the pyruvate and $\alpha$-ketoglutarate dehydrogenase complexes and the $\alpha$-ketoglutarate dehydrogenase component (EIkg) were measured spectrophotometrically according to Guest \& Creaghan (1973). Pyruvate dehydrogenase (EIp) was assayed together with pyruvate oxidase by the method used for Erkg but with pyruvate as substrate.

Antiserum. A young New Zealand white rabbit was injected subcutaneously, in the neck and shoulders, on alternate days for 12 days with $0.5 \mathrm{ml}$ of an $E$. coli в lipoamide dehydrogenase (I $\mathrm{mg}$ protein $/ \mathrm{ml} 0.03 \mathrm{M}$-potassium phosphate, $\mathrm{pH} 7 \cdot 6$, filter sterilized) mixed with an equal volume of Freund's incomplete adjuvant. After three weeks it received a similar course of 6 injections and was bled $\mathrm{r} 4, \mathrm{I} 7$ and 20 days after the last injection. From $60 \mathrm{ml}$ of serum the fraction containing antibody was concentrated threefold by precipitation with ammonium sulphate at $50 \%$ saturation followed by dialysis versus potassium phosphate buffer $(0.02 \mathrm{M}, \mathrm{pH} 7.4$ containing $0 . \mathrm{I} 4 \mathrm{M}-\mathrm{NaCl})$ and stored at $-20{ }^{\circ} \mathrm{C}$. In the standard test for antibody activity, purified lipoamide dehydrogenase ( $5 \mu \mathrm{g}$ protein) was incubated for $20 \mathrm{~min}$ at room temperature with graded amounts of antiserum in a final volume of $0.2 \mathrm{ml}$. After centrifuging $\left(20 \mathrm{~min}\right.$, $10000 \mathrm{~g}$ at $4{ }^{\circ} \mathrm{C}$ ) to remove precipitated antigen-antibody complex the lipoamide dehydrogenase activity remaining in the supernatant fluid was assayed. Under these conditions $4 \mu \mathrm{l}$ was the minimum volume of concentrated antiserum to completely inactivate $5 \mu \mathrm{g}$ of purified lipoamide dehydrogenase.

Immunodiffusion. Ouchterlony double diffusion was conducted on microscope slides $(\mathrm{I} \times 3$ in) in tris- $\mathrm{HCl}$ buffer $(5 \mathrm{mM}, \mathrm{pH} 7 \cdot 3)$ containing $\mathrm{NaCl}$ and sodium azide $(0.8$ and $0.2 \%, \mathrm{w} / \mathrm{v}$, respectively) and solidified with agarose $(0.9 \%, \mathrm{w} / \mathrm{v}$; Miles-Seravac). Wells ( $3 \mathrm{~mm}$ diam and $10 \mu \mathrm{l}$ vol.) were cut in a hexagonal pattern $8 \mathrm{~mm}$ distant from a similar 
centre well. Several dilutions of antiserum and antigen solutions were used and the most satisfactory results were obtained with Io $\mu \mathrm{l}$ volumes of a tenfold dilution of concentrated antiserum in the centre well and lipoamide dehydrogenase $(0.1 \mathrm{mg} / \mathrm{ml})$ or crude ultrasonic extracts of Escherichia coli (10 $\mathrm{mg}$ protein/ml) in the lateral wells. Gels were developed at room temperature for 24 to $48 \mathrm{~h}$, photographed with dark ground illumination and then washed in saline and water before drying and staining with Ponceau $\mathrm{S}$. The interpretation of precipitin patterns obtained with antisera specifically directed against pure proteins has been discussed by Gasser \& Gasser (1971).

Genetic methods. Conjugation experiments were performed by a cross-streak mating procedure (Herbert \& Guest, 1968). This procedure was modified slightly with strain Aw9 which had to be concentrated 50 -fold because of its relatively weak donor activity. Transductions were performed with phage PI (Guest, 1974) and transductants were selected on glucose minimal media containing appropriate supplements. In addition, $\mathrm{Lpd}^{+}$and $\mathrm{Ace}^{+} \mathrm{Lpd}^{+}$transductants were also selected on enriched acetate and enriched succinate media respectively. The distribution of non-selective markers was scored by replicaplating after purification of transductant colonies.

Materials. A sample of purified E. coli в lipoamide dehydrogenase was kindly provided by $\operatorname{Dr} \mathrm{C} . \mathrm{H}$. Williams, Jun. Its specific activity was initially $600(\mu \mathrm{mol} 3$-acetyl NAD reduced $/ \mathrm{mg}$ protein $/ \mathrm{h}$ ) when assayed by the procedure adopted in the present work.

\section{RESULTS}

\section{Immunological studies}

The antiserum raised against purified lipoamide dehydrogenase (lpdh) from Escherichia coli B gave single precipitin lines with this antigen and with an ultrasonic extract of wildtype E. coli KI2 (Fig. I, A and B). These lines were fused, indicating complete immunological identity between the lipoamide dehydrogenases of the two strains of $E$. coli. The crude extracts undoubtedly contain lpdh complexed with other components of both keto acid dehydrogenase complexes in addition to some in the uncomplexed state. However, multiple precipitin lines which might correspond to these different states of lpdh were not apparent under the conditions used for immunodiffusion. Immunodiffusion tests were conducted with extracts of each of the $l p d$ mutants using purified lpdh and wild-type extract in adjacent wells. Three types of reaction were observed and these are illustrated with representative mutants in Fig. I (C, D and F). Several mutants, lpd 3, 4, 5 and 9, were CRM + because they gave reactions identical to the wild-type, indicating the presence of cross-reacting mutant proteins (Fig. I D). These included the three $l p d$ mutants ( 4,5 and 9 ) which exhibited approximately 10 \% of wild-type lpdh activity (Guest \& Creaghan, 1973). Another group of mutants $(\mathrm{CRM}-)$ produced no detectable reaction with the antiserum; these were lpd6, 8 and IO (Fig. I, C). With the third group, consisting of mutants $l p d 2$ and 7 , the precipitin lines for mutant and wild-type antigens were only partially fused and this was accompanied by spur formation (Fig. I, F). This reaction of partial identity indicates that the mutant proteins have some but not all of the antigenic sites of the wild-type protein. The corresponding antibodies can therefore diffuse past the mutant precipitin line and react with the adjacent wild-type antigen to form the spurs. The remaining mutant, $l p d I$, was difficult to classify but probably represented an extreme example of this third category because it exhibited a very weak and diffuse line of identity between two substantial spurs. Several other strains were tested, e.g. aceE (A2T3), sucA (WGAsucA26) and a mutant H2, isolated 


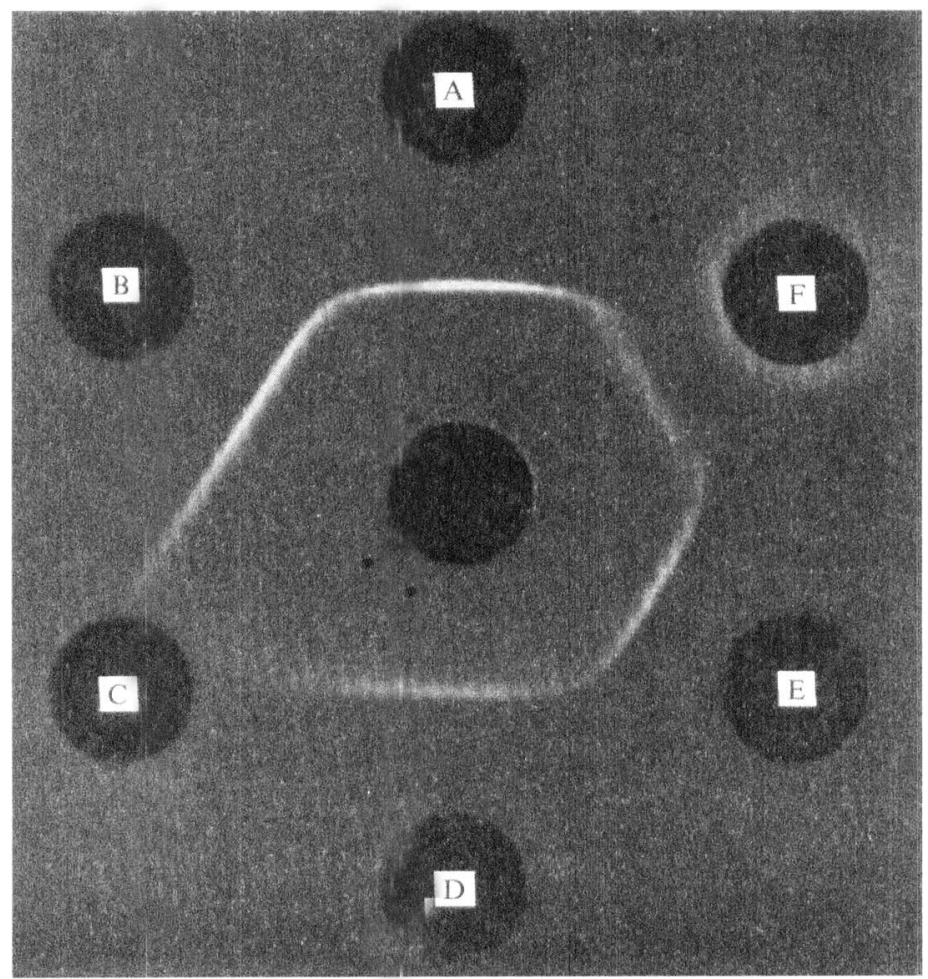

Fig. I. Immunodiffusion in agarose with antisera in the centre well, and lateral wells containing: $\mathrm{I} \mu \mathrm{g}$ of lipoamide dehydrogenase purified from $E$. coli $\mathrm{B}(\mathrm{A})$; ultrasonic extract equivalent to 100 $\mu \mathrm{g}$ protein of E. coli KI2 WGA (B and E); H/pdIo (C); WGAlpd5 (D); and H/pd7 (F).

with the $l p d$ mutants and tentatively identified as an $a c e E s u c B$ double mutant. They were all $\mathrm{CRM}+$, which is consistent with their $\mathrm{Lpd}^{+}$phenotype. Another mutant $\mathrm{H}_{7} \mathrm{~B}$, probably an ace, lpd double mutant, was CRM - and the double amber mutant WGAaceE64sucA35 gave a weak CRM + reaction which is consistent with a limited expression of its wild-type lpd gene (Creaghan \& Guest, 1972). No cross-reaction was observed with purified pig heart lpdh (Sigma). Likewise, antibodies raised against the mammalian enzyme do not precipitate the bacterial enzyme (Hayakawa, Aikawa, Otsuka \& Koike, 1967).

The effect of lpdh antibodies on the activities of the overall pyruvate and $\alpha$-ketoglutarate dehydrogenase complexes, the dehydrogenase component ( $\left.E_{1}\right)$ and lpdh (E3) in an ultrasonic extract of strain WGA was also investigated (Fig. 2). As in the standard test for antibody titration, antigen-antibody complexes were removed by sedimentation because they retained enzymic activity. These titrations indicated that the lpdh activities of crude extracts and the purified enzyme were equally sensitive to the antiserum. It can then be calculated that lpdh represents approximately 0.3 to $0.4 \%$ of the total protein in the extract. The precipitation of lpdh activity was paralleled exactly by loss of overall pyruvate complex activity, whereas inactivation of the $\alpha$-ketoglutarate dehydrogenase complex preceded the disappearance of lpdh. This could be due to a failure to detect low activities of the $\alpha$-ketoglutarate complex, or alternatively it could indicate that the E3 component has a lower affinity for this complex. It is interesting to note that the EI components of both complexes also appear to be precipitated with the antiserum. This is presumably a secondary effect stemming from a primary interaction between lpdh and its antibodies and the mutual 


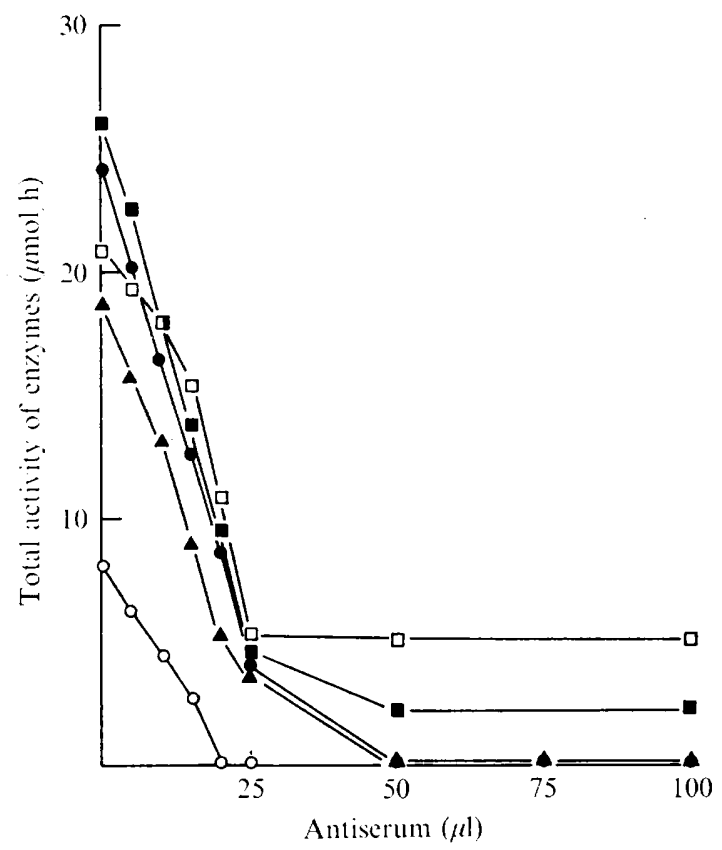

Fig. 2. The effect of lipoamide dehydrogenase antiserum on the activities of $\alpha$-keto acid dehydrogenase complexes and their components in an ultrasonic extract of Escherichia coli KI 2 strain wGA. Equivalent samples of extract containing $8.5 \mathrm{mg}$ protein were incubated with antiserum in buffered saline and centrifuged according to the standard procedure (see Methods) before assaying the enzyme activities remaining in the supernatant fluid: $\Delta$, lipoamide dehydrogenase $\left(E_{3}\right)$; $\bullet$, overall pyruvate dehydrogenase complex; $O$, overall $\alpha$-ketoglutarate dehydrogenase complex; $\square$, pyruvate dehydrogenase (EIp) plus pyruvate oxidase; $\square$, $\alpha$-ketoglutarate dehydrogenase (EIkg).

affinities of the Er and E3 components for the core component E2, which results in coprecipitation of all components. The residual EI kg activity could represent excess synthesis of this component but it may be simply due to exclusion of this component (or EI-E2 partial complex) by the antibody. The same may be true for the EIp component, where an 'excess' of this component is known to be present in the complex (Vogel, Hoehn \& Henning, I972), but the activity could in addition be due to the flavoprotein pyruvate oxidase which also catalyses the reduction of ferricyanide in the assay used.

\section{Genetic studies}

The chromosomal location of the $l p d$ gene has been indicated by a detailed investigation with one strain T3A58lpdI (Guest, 1974). Further studies with a total of ro lpd mutants are now reported.

Conjugation. The positions of all ten $l p d$ mutations were restricted to the I to 9 minute region of the $E$. coli linkage map by cross-streak conjugation tests using several Hfr and F-prime donors (Fig. 3). In each cross $\mathrm{Lpd}^{+}$recombinants were selected on glucose, succinate and acetate minimal media and the donors were contra-selected nutritionally or with streptomycin. $\mathrm{Lpd}^{+}$recombinants were obtained with $\mathrm{H}, \mathrm{R} 4$ and KLF4 as donors but not with BII or KLFI; with $F_{2}$-gal a weak positive result was observed, probably due to chromosomal mobilization. Strains containing the markers shown in Fig. 3 were used to check the characteristics of the donor strains. 


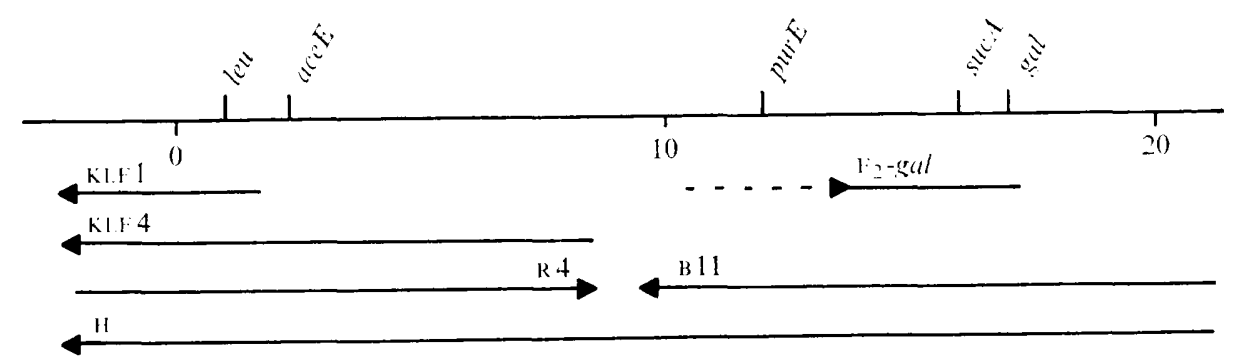

Fig. 3. Segment of the linkage map of Escherichia coli KI 2 showing the positions of standard markers used in conjugation studies, the points of origins of $\mathbf{H f r}$ and F-prime strains and the approximate chromosomal regions carried by the F-prime factors.

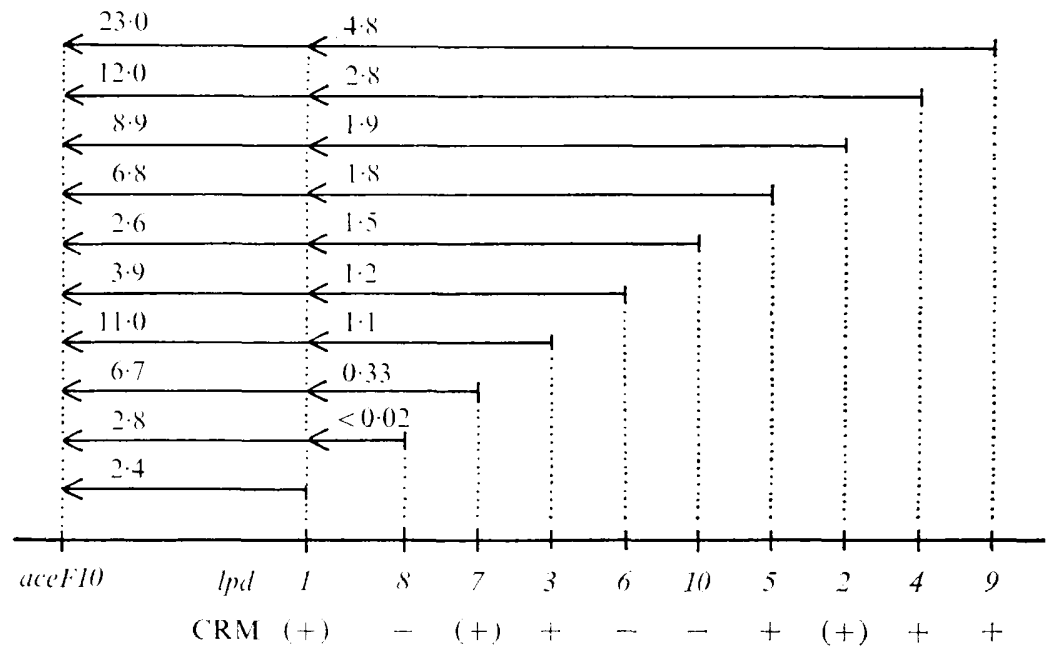

Fig. 4. Genetic map showing the likely positions of $l p d$ mutant loci relative to the aceFIo and $l p d I$ sites. The map distances correspond to the frequencies of $\mathrm{Ace}^{+} \mathrm{Lpd}^{+}$or $\mathrm{Lpd}^{+}$transduction expressed as a percentage of the frequency of $\mathrm{Nad}^{+}$transduction in the same cross when the lpd mutants served as donors with aceFIonadC and $l p d i n a d C$ recipients. The orientation of each mutant locus relative to aceFIO and $l p d r$ is based on the distribution of the non-selective Nad marker in the Ace ${ }^{+}$ $\mathrm{Lpd}^{+}$and $\mathrm{Lpd}^{+}$transductants. Also shown are the immunological characteristics of the mutants: $\mathrm{CRM}+$, cross-reacting material present and indistinguishable from wild-type; $\mathrm{CRM}(+)$, present but exhibiting only partial identity with wild-type; CRM-, no cross-reacting material detected.

Three-factor transductional analysis. Preliminary two-factor crosses with phage PI lysates of the $l p d$ mutants indicated that all ten $l p d$ sites were linked to $l e u$ (cotransduction frequencies of between 8 and $25 \%$ were obtained when limited numbers of Leu ${ }^{+}$transductants were examined during the construction of the WGAslpd strains, see Methods). The results of a more detailed three-factor analysis, designed to determine the positions of the $l p d$ mutations with respect to the aceFIo and $l p d I$ sites are summarized in Fig. 4. For this purpose PIlysates prepared by two successive lytic cycles on lpd donors were used with recipients AIonadC(PI) (nadC, aceFIo) and T3A58lpdrnadC (nadC, lpdI) in turn. The frequencies of $\mathrm{Ace}^{+} \mathrm{Lpd}^{+}$or $\mathrm{Lpd}^{+}$and $\mathrm{Nad}^{+}$transductions were determined in each cross and the former $\left(\mathrm{Ace}^{+} \mathrm{Lpd}^{+}\right.$or $\mathrm{Lpd}^{+}$) were expressed as a percentage of the corresponding frequency of $\mathrm{Nad}^{+}$transduction, which ranged from 6 to 30 transductants/10 ${ }^{6}$ PI particles in different experiments. These recombination frequencies (map distances) all indicated close linkage between each $l p d$ marker and the standard aceFıo and $l p d I$ sites. Appropriate controls to 


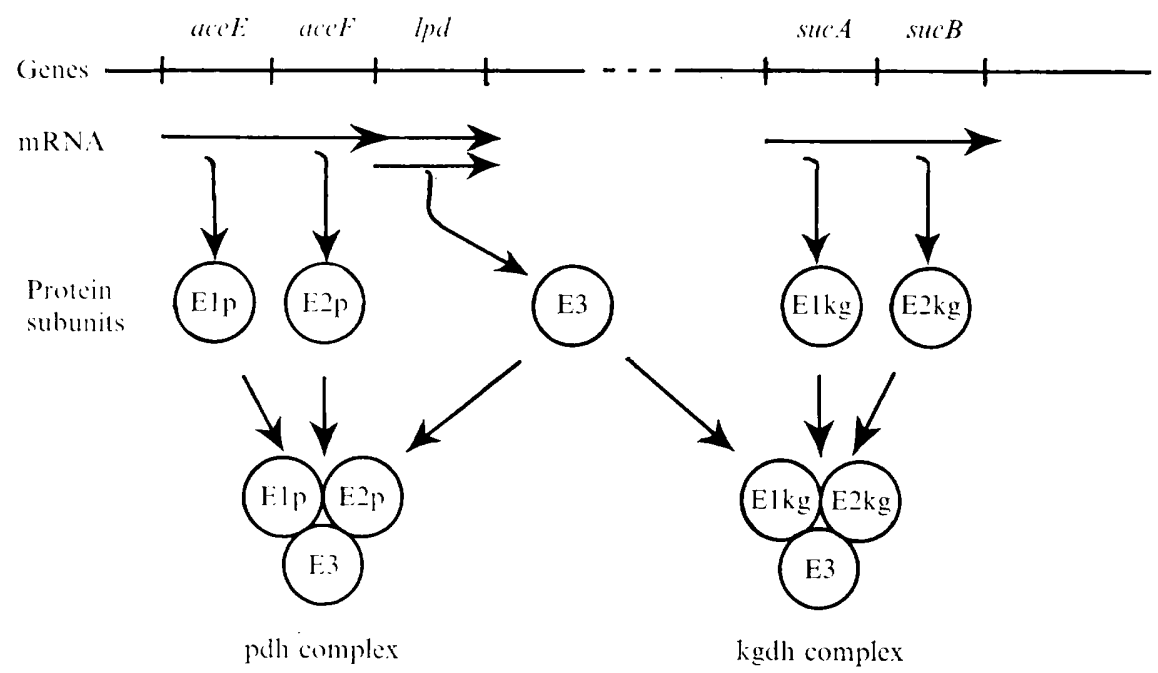

Fig. 5. Diagrammatic representation of the gene-protein relationships of the $\alpha$-keto acid dehydrogenase complexes of Escherichia coli. The polarities of ace and suc in RNA transcription and possible mechanisms for lpd transcription (Guest, 1974) are indicated. (Each complex contains multiple copies of each subunit rather than the one shown: pdh = pyruvate dehydrogenase; kgdh $=\alpha$-ketoglutarate dehydrogenase).

monitor reversion were included and these were negative. The segregation of the donor $\mathrm{Nad}^{+}$ marker was also examined with about $60 \mathrm{Ace}^{+} \mathrm{Lpd}^{+}$or $\mathrm{Lpd}^{+}$transductants from each cross. An average of $60 \%$ (range 46 to $74 \%$ ) were $\mathrm{Nad}^{+}$, indicating that all the $l p d$ sites are to the right of both standard markers. Distributions of less than $18 \% \mathrm{Nad}^{+}$would have indicated the reverse order (Guest, 1974). The positions of the $l p d 2$ to $I 0$ mutations relative to each other are based solely on recombination frequencies with $l p d I$, so the order shown in Fig. 4 is by no means established. In fact, a somewhat different order would be obtained if the $l p d$ to aceFro distances had been used. Abortive transduction was observed in all the crosses involving the $a c e F$ recipient but never between $l p d r$ and the other lpd mutants, which indicates that the ten $l p d$ mutants belong to a single functional unit.

Suppression. The suppressibility of the lpd mutants was tested with three different amber suppressors which insert serine (supD), tyrosine (supF) and glutamine (sup $U$ ) in response to the amber codon. For this purpose the $l p d$ mutations were introduced into a streptomycinresistant derivative of strain WGA which contains a trpA-amber mutation and provides an independent test for suppression. Cross-streak conjugation tests with an FI4supU donor failed to produce Sup ${ }^{+}$recombinants on several media selective for the $\mathrm{Lpd}^{+}$phenotype. By contrast, tryptophan-independent recombinants were obtained with all of the wGAslpd strains. In parallel studies using trp-deletion strains carrying sup D or $F$ as donors, tryptophanindependent transductants (which could arise only by suppression) were selected and scored for the unselected Lpd phenotype. In no case was there any evidence for suppression of the lpd mutations, although control tests with WGAsucA36-amber (Creaghan \& Guest, 1972) were positive. It can thus be concluded that none of the $l p d$ mutations is sensitive to any of these three amber suppressors. 


\section{DISCUSSION}

The results show that all of the ten independent mutations leading to a deficiency in lipoamide dehydrogenase activity are located in one small segment of the $E$. coli chromosome. The failure to detect abortive transduction between pairs of mutants indicates that a single functional unit is affected. Furthermore, the presence of lipoamide dehydrogenase crossreacting material in extracts of at least six of the mutants indicates that they possess missense mutations in the lipoamide dehydrogenase structural gene, $l p d$. The results confirm and extend previous findings (Guest \& Creaghan, 1972; 1973; Guest, 1974; Alwine et al. 1973) and show that the lpdh components (E3) of the pyruvate and $\alpha$-ketoglutarate dehydrogenase complexes are specified by a single gene $(l p d)$ which is closely-linked to the structural genes for the other components of the pyruvate dehydrogenase complex in the following order: aceE-aceF-lpd. This conclusion is illustrated in Fig. 5 which summarizes the gene-protein relationships of $\alpha$-keto acid dehydrogenase complexes. The organization of these genes raises interesting questions concerning their evolution and the regulation of their expression, particularly the regulation of $l p d$ expression relative to the other genes. Possible mechanisms for the latter have been discussed (Guest, 1974) and they depend to a large extent on the nature of the region between $a c e F$ and $l p d$. The cotransduction frequency measured with aceFIo and $l p d I$, the closest CRM + lpd mutant, was $97 \%$ and values of $3.3, \mathrm{I} \cdot 7, \mathrm{I} \cdot 9, \mathrm{I} \cdot 3$ and $2.4 \%$ (average $2 \cdot \mathrm{I} \%$ ) have been obtained for the recombination frequency between the same two sites (Guest, 1974). Using the mapping function of Wu (I966) to relate cotransduction frequencies to map distances in time units, the value of $97 \%$ corresponds to 0.02 min (assuming the length of a PI transducing fragment is 2.0 min). Then, if there are approximately 3000 'average genes' each containing 1000 base pairs in the 90 min linkage map (Taylor, 1970), the aceFIo-lpdI distance corresponds to approximately 0.67 of an 'average gene'. An alternative approach to evaluating this distance comes from the estimated recombination frequency of $4.2 \%$ between the extremities of the tryptophan synthetase $A$-gene, which corresponds approximately to 800 base pairs (Yanofsky, Drapeau, Guest \& Carlton, 1967). This would indicate for aceFIo to $l p d I$ a separation of approximately 400 base pairs or 0.4 of an 'average gene'. Since the aceFIo site is probably some distance from the distal end of the $a c e F$ gene (Henning \& Hertz, 1964) the actual distance between the two genes may be significantly less than estimated values. These considerations lead therefore to the conclusion that the $a c e F$ and $l p d$ genes are contiguous or separated by a relatively short regulatory region but not by any other gene(s).

We wish to thank Dr C. H. Williams, Jun. for a sample of purified lipoamide dehydrogenase and Professor J. K. A. Beverley and Dr T. Drabble for advice and assistance with the immunological techniques.

\section{REFERENCES}

Alwine, J. C., Russell, F. M. \& Murray, K. N. (1973). Characterization of an Escherichia coli mutant deficient in dihydrolipoyl dehydrogenase activity. Journal of Bacteriology I15, I-8.

Creaghan, I. T. \& Guest, J. R. (1972). Amber mutants of the $\alpha$-ketoglutarate dehydrogenase gene of Escherichia coli $\mathrm{KI}$ 2. Journal of General Microbiology 7I, 207-220.

FlatgaARd, J. E., Hoehn, B. \& HenNing, U. (197I). Mutants of Escherichia coli K-I 2 which synthesize the pyruvate dehydrogenase complex constitutively. Archives of Biochemistry and Biophysics r43, 46I-470.

Gasser, F. \& GaSSER, C. (197I). Immunological relationships among lactic dehydrogenases in the genera Lactobacillus and Leuconostoc. Journal of Bacteriology 106, I I3-I25.

GUEST, J. R. (I974). Gene-protein relationships of the $\alpha$-keto acid dehydrogenase complexes of Escherichia coli K12: chromosomal location of the lipoamide dehydrogenase gene. Journal of General Microbiology 8o, 523-532. 
Guest, J. R. \& CREAGHAN, I. T. (I972). Lipoamide dehydrogenase mutants of Escherichia coli K12. Biochemical Journal r3o, 8 P.

Guest, J. R. \& Creaghan, I. T. (1973). Gene-protein relationship of the $\alpha$-keto acid dehydrogenase complexes of Escherichia coli KI2: isolation and characterization of lipoamide dehydrogenase mutants. Journal of General Microbiology 73, 197-210.

Hayakawa, T., Aikawa, T., Otsuka, K. \& Kolke, M. (1967). Immunochemical relationships among pig heart lipoamide dehydrogenases. Journal of Biochemistry 62, 396-397.

Henning, U., Dennert, G., Hertel, R. \& Shipp, E. S. (I966). Translation of the structural genes of the E. coli pyruvate dehydrogenase complex. Cold Spring Harbor Symposia for Quantitative Biology 3r, 227-234.

Henning, U. \& Hertz, C. (1964). Ein Strukturgen-Komplex für den Pyruvat-Dehydrogenase-Komplex von Escherichia coli $\mathrm{K} 12$. Zeitschrift für Vererbungslehre 95, 260-275.

Herbert, A. A. \& Guest, J. R. (I968). Biochemical and genetic studies with lysine+methionine mutants of Escherichia coli: lipoic acid and $\alpha$-ketoglutarate dehydrogenase-less mutants. Journal of General Microbiology 53, 363-38I.

Herbert, A. A. \& Guest, J. R. (1969). Studies with $\alpha$-ketoglutarate dehydrogenase mutants of Escherichia coli. Molecular and General Genetics 105, I82-190.

TAYLOR, A. L. (1970). Current linkage map of Escherichia coli. Bacteriological Reviews 34, I55-175.

Vogel, O., Hoenn, B. \& Henning, U. (1972). Molecular structure of the pyruvate dehydrogenase complex from Escherichia coli K-I2. Proceedings of the National Academy of Sciences of the United States of America 69, 16I5-16I9.

WU, T. T. (1966). A model for three-point analysis of random general transduction. Genetics 54, 405-4I0.

Yanofsky, C., Drapeau, G. R., Guest, J. R. \& Carlton, B. C. (1967). The complete amino acid sequence of the tryptophan synthetase A protein ( $\alpha$ subunit) and its colinear relationship with the genetic map of the A gene. Proceedings of the National Academy of Sciences of the United States of America 57, $296-298$. 